\title{
Bivariate Interpolation with Quadratic Box Splines
}

\section{By Morten Dæhlen and Tom Lyche}

\begin{abstract}
Existence and uniqueness results are given for interpolation with translates of a bivariate, three-directional, $C^{0}$-quadratic box spline over a finite polygonal region. A Hermite interpolation problem for a slightly more general box spline is also considered.
\end{abstract}

1. Introduction. Recently, existence and uniqueness questions for multivariate interpolation have received considerable attention. See [2], [3], [8], and references therein. In general, we are given an $n$-dimensional space of functions

$$
S=\operatorname{span}\left\{\phi_{1}, \ldots, \phi_{n}\right\}
$$

on a region $\Omega$ in $R^{s}$. The Lagrange interpolation problem is to determine a subset $P$ of $\Omega$ such that the $n$ by $n$ collocation matrix with elements $\phi_{j}\left(x^{i}\right)$ is nonsingular for any choice of distinct $x^{1}, \ldots, x^{n}$ in $P$. We refer to this as a unisolvence problem.

We are interested in a box spline unisolvence problem. Specifically, in this paper, the $\phi_{j}$ 's will be translates of one bivariate $(s=2)$ box spline on a uniform 3-direction (type 1) grid.

Given two linearly independent vectors $c^{1}$ and $c^{2}$ in $R^{2}$, a uniform 3-direction grid is constructed by drawing straight lines in the three directions $c^{1}, c^{2}$ and $c^{1}+c^{2}$ through all points in $R^{2}$ of the form $j c^{1}+k c^{2}, j, k \in Z$. We will only consider the standard grid $G$ obtained by choosing $c^{1}=d^{1}=(1,0)^{T}$ and $c^{2}=d^{2}=(0,1)^{T}$. (We can map any grid into the standard one by mapping $c^{1}$ into $d^{1}$ and $c^{2}$ into $d^{2}$.)

The region $\Omega$ will be a bounded, convex set as shown in Figure 1.1. We obtain any such $\Omega$ by removing two triangles of size $k_{l}$ and $k_{u}$ from the lower right and upper left corner of a rectangle of size $n_{1}, n_{2} . \Omega$ is a triangle if $n_{1}=n_{2}$ and $k_{l}=0$, $k_{u}=n_{2}$ or $k_{u}=0, k_{l}=n_{1}$. Similarly, we obtain a trapezoid, a pentagon, or a hexagon depending on the values of $k_{l}, k_{u}, n_{1}$, and $n_{2}$. We assume that $n_{1}>0$, $n_{2}>0$, and $0 \leq k_{l}, k_{u} \leq \min \left\{n_{1}, n_{2}\right\}$.

The box splines of interest in this paper are piecewise polynomials on $G$. Given three integers $m=\left(m_{1}, m_{2}, m_{3}\right)$ with $m_{1}>0, m_{2}>0$, and $m_{3} \geq 0$, we can use the following simple definition

$$
B^{\left(m_{1}, m_{2}, m_{3}\right)}(x, y)= \begin{cases}M_{m_{1}}(x) M_{m_{2}}(y) & \text { if } m_{3}=0 \\ \int_{0}^{1} B^{\left(m_{1}, m_{2}, m_{3}-1\right)}(x-t, y-t) d t & \text { otherwise. }\end{cases}
$$

Here, $M_{k}(t)=M_{k}(t \mid 0,1, \ldots, k)$ is the univariate B-spline of order $k$ with knots $0,1, \ldots, k$ normalized to have integral equal to one. Thus, $B^{m}$ is a tensor product B-spline if $m_{3}=0$.

Received June 8, 1987.

1980 Mathematics Subject Classification (1985 Revision). Primary 41A05, 41A63; Secondary $65 \mathrm{D} 05$.

Key words and phrases. Interpolation, box splines, bivariate, three-direction grid. 


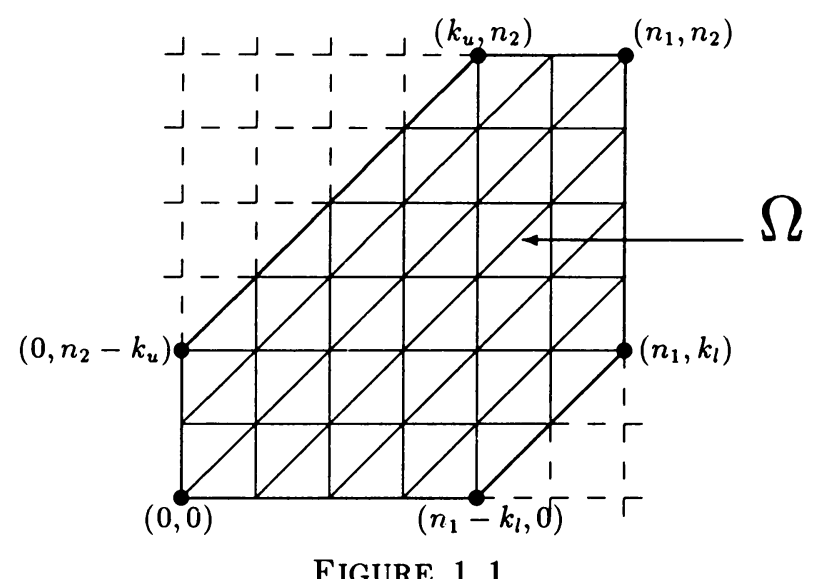

A bounded, polygonal, convex set $\Omega$ with boundary along a 3-direction grid
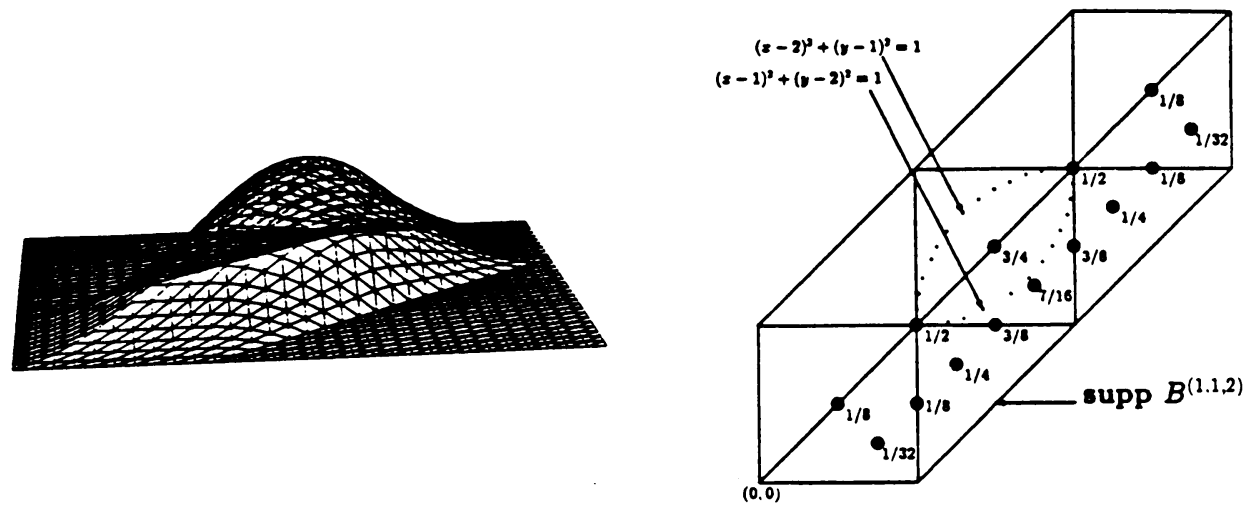

FIGURE 1.2

The $C^{0}$-quadratic $(1,1,2)$ box spline (left). The support, together with selected function values along the diagonals $y=x$ and $y=x-1 / 2$, are given on the right half of the figure.

The relation (1.1) defines $B^{m}$ for every point in $R^{2}$. If we define the univariate B-splines to be right-continuous, i.e., we set

$$
M_{1}(t)=\chi_{[0,1)}(t)= \begin{cases}1 & \text { if } 0 \leq t<1 \\ 0 & \text { otherwise }\end{cases}
$$

then the value of $B^{m}$ or one of its derivatives on a grid line is obtained as the limit from the right in the $x$ direction and from above in the $y$ direction.

We recall [1] some simple properties of $B^{m}$. The box spline $B^{m}$ is a polynomial of degree $|m|-2$ on each triangle in $G$, where

$$
|m|=m_{1}+m_{2}+m_{3} .
$$

The support of $B^{m}$ is a six-sided polygon as shown in Figure 1.2 for the $(1,1,2)$ case. Moreover, partial derivatives of order $\leq|m|-2-\max m_{j}$ are continuous everywhere. 
The outline of this paper is as follows. In Section 2 we give a convenient formula for the number of box splines which are nonzero on the interior of the region $\Omega$ shown in Figure 1.1. Since these functions are linearly independent on $\Omega$ [1], this number is the dimension of the space $S$. In Section 3 we study the unisolvence problem for the quadratic box spline $B^{(1,1,2)}$. Similar problems have been studied in [4] (linear nonuniform) and [5] ( $C^{1}$-quadratic on a 4-direction grid). In Section 4 we consider a Hermite interpolation problem for the box spline $(1,1, k)$. We conclude the paper with an example.

$C^{0}$-quadratic box splines have been used in [9] to model objects with slope discontinuities along diagonals. See also [6].

For results on cardinal interpolation with box splines, see [10], [7] and references therein. Since $\Omega$ is a finite region, these results are not directly applicable.

2. The Spline Space. Let $\Omega$ be as in Figure 1.1. We are interested in the space

$$
S_{m}(\Omega)=\left\{\sum_{(i, j) \in I(\Omega)} c_{i, j} B_{i, j}^{m}: c_{i, j} \in R\right\}
$$

where

$$
B_{i, j}^{m}(x, y)=B^{m}(x-i, y-j), \quad x, y \in R
$$

and

$$
I(\Omega)=\left\{(i, j) \in Z^{2}: B_{i, j}^{m}(x, y) \neq 0 \text { for some }(x, y) \in \Omega^{0}\right\} .
$$

Here, $\Omega^{0}$ is the interior of the set $\Omega$. We recall [1] that for all $(x, y) \in \Omega^{0}$ and any $m$ we have

$$
\sum_{(i, j) \in Z^{2}} B_{i, j}^{m}(x, y) \equiv 1
$$

moreover, for all $(i, j) \in Z^{2}$,

$$
\int_{R^{2}} B_{i, j}^{m}(x, y) d x d y=1
$$

It is convenient to indicate the location of the points in the index set $I(\Omega)$ by drawing its convex hull $[I(\Omega)]$. The set $[I(\Omega)]$ is a six-sided polygon as shown in Figure 2.1. The reader can verify this by tracing the location of the lower left corner of the support of $B^{m}$ as one moves $B^{m}$ along the boundary of $\Omega$.

A convenient formula for the number of elements $|I(\Omega)|$ in $I(\Omega)$ is given by the following result.

PROPOSITION 2.1. Suppose $\Omega \subset R^{2}$ is of the form indicated in Figure 1.1. Then

$$
|I(\Omega)|=\mu+\left(m_{1}-1\right) h+\left(m_{2}-1\right) v+\left(m_{3}-1\right) d+g,
$$




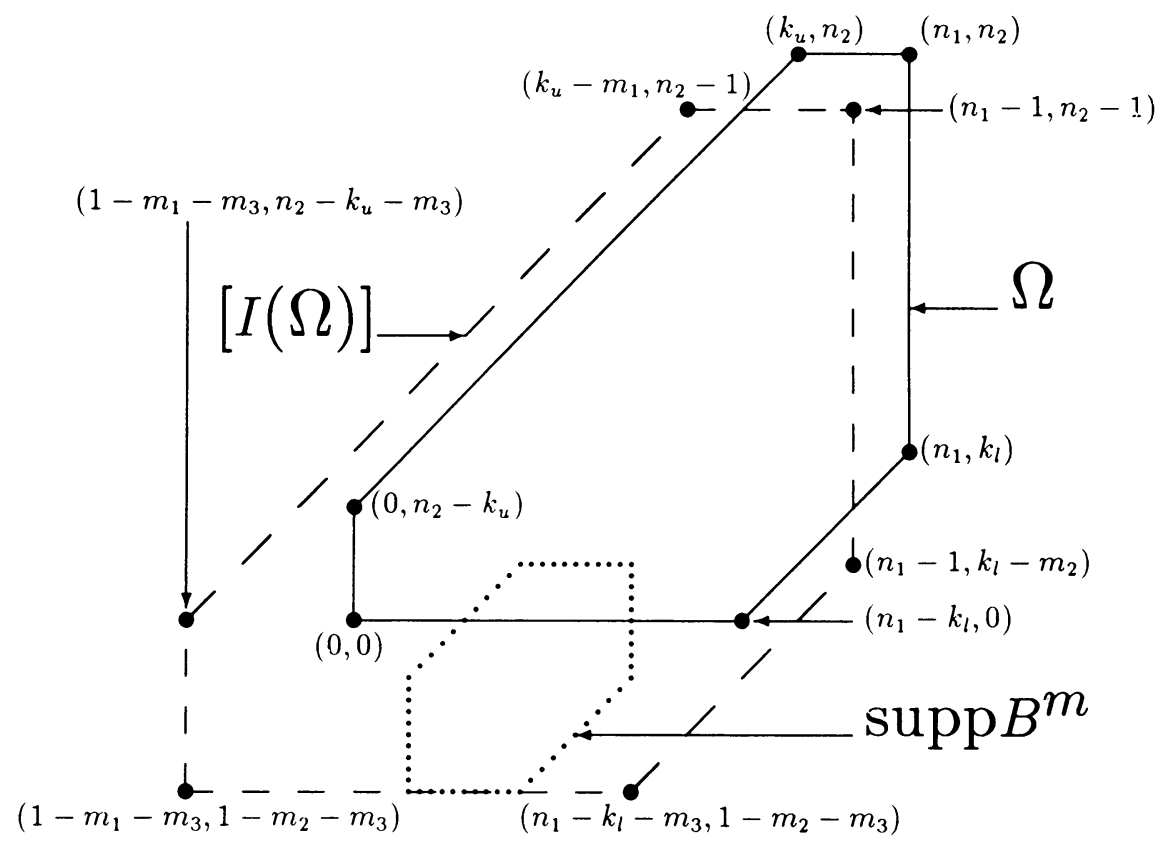

FIGURE 2.1

$\Omega($ solid $),\left[I_{m}(\Omega)\right]$ (dashed), and the support of $B^{m}$ (dotted).

where $h, v$ and $d$ are respectively the number of horizontal, vertical and diagonal lines in $G \cap \Omega$, and $g$ the number of grid points. More precisely,

$$
\begin{aligned}
\mu & =\left(m_{1}-1\right)\left(m_{2}-1\right)+\left(m_{1}-1\right)\left(m_{3}-1\right)+\left(m_{2}-1\right)\left(m_{3}-1\right), \\
h & =n_{2}+1, \\
v & =n_{1}+1, \\
d & =n_{1}+n_{2}-k_{l}-k_{u}+1, \\
g & =\left(n_{1}+1\right)\left(n_{2}+1\right)-\frac{1}{2} k_{l}\left(k_{l}+1\right)-\frac{1}{2} k_{u}\left(k_{u}+1\right) .
\end{aligned}
$$

Proof. $|I(\Omega)|$ is equal to the number of points in a rectangle with two corners cut off. In particular (cf. Figure 2.1), we have

$$
\begin{aligned}
|I(\Omega)|= & \left(n_{1}+m_{1}+m_{3}-1\right)\left(n_{2}+m_{2}+m_{3}-1\right) \\
& -\frac{1}{2}\left(k_{l}+m_{3}-1\right)\left(k_{l}+m_{3}\right)-\frac{1}{2}\left(k_{u}+m_{3}-1\right)\left(k_{u}+m_{3}\right) .
\end{aligned}
$$

A simple rearrangement gives (2.4).

As already mentioned, the box splines used in (2.1) are linearly independent. Thus $n=|I(\Omega)|$ is the dimension of the space $S_{m}(\Omega)$.

It is of interest to consider the ordering of basis functions and interpolation points. A natural way would be to pick one of the three directions, say, the diagonal direction, and order the points in $I(\Omega)$ according to which diagonal the lower left corner of the support of the box spline lies on. There are

$$
\tilde{d}=d+m_{1}-1+m_{2}-1
$$

diagonals in $I(\Omega)$, where $d$ is the number of diagonals in $\Omega$.

We could start with all the points on the lower right diagonal moving from bottom left to top right along the diagonal. Then we could continue with each of 
the diagonals above in the same way. We call this the $d$-ordering of $I(\Omega)$. We define $h$-ordering and $v$-ordering in a similar way.

Suppose now that the box splines have been ordered in some order $B_{1}, B_{2}, \ldots$, $B_{n}$. The next problem is to order the interpolation points $x^{1}, x^{2}, \ldots, x^{n}$. How this should be done depends on the purpose. To limit the band width of the matrix with elements $B_{j}\left(x^{i}\right)$, one should order the $x^{i}$ 's in a similar way as the $B_{j}$ 's.

Suppose the $d$-ordering is used for the functions. If $\tilde{d}>d$, it is not clear how to choose a $d$-ordering for the points. One way could be as follows. We first take all points which are at least as close to the lower right diagonal of $\Omega$ as to any other diagonal in $\Omega$. One would then take all points which are closer to the second diagonal in $\Omega$ and at least as close to the second diagonal as to the third diagonal. Continuing in this way, we divide the points according to which diagonal strip they belong to. To order the points within one strip, suppose $p^{1}$ and $p^{2}$ are two points in the same diagonal strip and let $\nu_{1}$ and $\nu_{2}$ be normals to the diagonal passing through $p^{1}$ and $p^{2}$, respectively. Then $p^{1}$ comes before $p^{2}$ if $\nu_{1}$ is below $\nu_{2}$. If $p^{1}$ and $p^{2}$ both lie on the same normal, then the point closest to the diagonal would be counted first.

We end this section by stating two convenient symmetry properties of box splines $B^{m}$ on a 3 -direction grid. For all $x, y \in R$

$$
\begin{gathered}
B^{m}(x, y)=\hat{B}^{m}\left(m_{1}+m_{3}-x, m_{2}+m_{3}-y\right), \\
B^{m}(x, y)=B^{m}(y, x) \quad \text { if } m_{1}=m_{2} .
\end{gathered}
$$

Here, $\hat{B}^{m}$ is the left-continuous version of $B^{m}$, i.e., we use left-continuous univariate B-splines $\hat{M}_{k}$ (for $\left.k=1, \hat{M}_{1}=\chi_{(0,1]}\right)$ in (1.1). The relations $(2.5)$ and (2.6) follow from (1.1) using induction on $m_{3}$. Relation (2.6) is trivial for $m_{3}=0$, and relation $(2.5)$, for $m_{3}=0$, follows from the fact that for all $t$ and $k, M_{k}(t \mid 0,1, \ldots, k)=$ $\hat{M}_{k}(k-t \mid 0,1, \ldots, k)$.

3. $C^{0}$-Quadratic Box Splines. We consider now the box spline unisolvence problem using the $m=(1,1,2)$ box spline. This box spline is shown in Figure 1.2. It is a piecewise quadratic function whose support consists of 10 triangles. These triangles together with a sample of function values are also shown in Figure 1.2. The box spline $B=B^{(1,1,2)}$ is continuously differentiable everywhere except across diagonals.

Example 3.1. Consider the unisolvence problem on the unit square $\Omega=[0,1]^{2}$ in $R^{2}$. By (2.4) we have $|I(\Omega)|=d+g=3+4=7$ box splines overlapping $\Omega$. The location of the lower left corner of the seven $(1,1,2)$ box splines overlapping the unit square is shown in Figure 3.1.

Let $f \in S_{m}(\Omega)$ be given by

$$
f=\left(B_{1}+B_{2}+2 B_{3}-2 B_{4}+2 B_{5}+B_{6}+B_{7}\right) / 2 .
$$

Using (2.6) and the explicit values in Table 3.1, it is easily shown that $f(y, x)=$ $f(x, y)$ and for $y \geq x$ that

$$
f(x, y)=(x-3 / 4)^{2}+(y-1 / 4)^{2}-10 / 16 .
$$


[7]

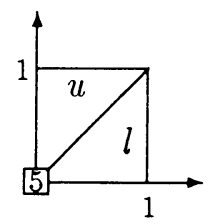

[6] [4]

[3] [1]

FIGURE 3.1

The d-ordering of the seven nonzero $(1,1,2)$ box splines

on the unit square.

TABLE 3.1

Explicit values of the seven $(1,1,2)$ box splines on the two triangles $l$ and $u$ in Figure 3.1 .

\begin{tabular}{ccc}
\hline Box spline & Value on $l$ & Value on $u$ \\
\hline 1 & $\frac{1}{2}(y-1)^{2}-\frac{1}{2}(x-1)^{2}$ & 0 \\
\hline 2 & $\frac{1}{2} x^{2}-\frac{1}{2} y^{2}$ & 0 \\
\hline 3 & $\frac{1}{2}(x-1)^{2}$ & $\frac{1}{2}(y-1)^{2}$ \\
\hline 4 & $1-\frac{1}{2} x^{2}-\frac{1}{2}(y-1)^{2}$ & $1-\frac{1}{2} y^{2}-\frac{1}{2}(x-1)^{2}$ \\
\hline 5 & $\frac{1}{2} y^{2}$ & $\frac{1}{2} x^{2}$ \\
\hline 6 & 0 & $\frac{1}{2}(x-1)^{2}-\frac{1}{2}(y-1)^{2}$ \\
\hline 7 & 0 & $\frac{1}{2} y^{2}-\frac{1}{2} x^{2}$ \\
\hline
\end{tabular}

It follows that $f$ vanishes identically on two circular $\operatorname{arcs} C_{l}$ and $C_{u}$ in $\Omega$. Thus, if $x^{1}, x^{2}, \ldots, x^{7}$ are chosen on $C_{l} \cup C_{u}$, say,

$$
\left\{x^{1}, \ldots, x^{7}\right\}=\left\{\left(\frac{1}{2}, 0\right),\left(1, \frac{1}{2}\right),(0,0),\left(0, \frac{1}{2}\right),\left(\frac{1}{2}, 1\right),(1,1),(u, v)\right\},
$$

where $(u, v) \in C_{l} \cup C_{u}$, then the matrix with elements $B_{j}\left(x^{i}\right)$ will be singular and the problem is not unisolvent.

This example shows that it would be very hard, in general, to give necessary and sufficient conditions for unisolvence. Let us now restrict our attention to interpolation at grid points $(i, j) \in Z^{2}$. By $(2.4)$ we have

$$
\operatorname{dim} S_{(1,1,2)}(\Omega)=g+d,
$$

where $g$ is the number of grid points and $d$ the number of diagonals in $\Omega$. Thus, in addition to interpolation at the grid points we need to specify $d$ interpolation conditions somehow. The next example shows that these points have to be chosen with some care.

Example 3.2. Suppose $\Omega$ is the hexagonal domain shown in Figure 3.2. This $\Omega$ has $g=7$ grid points and $d=3$ diagonals. Thus, $\operatorname{dim} S_{m}(\Omega)=10$. Let $x^{1}, x^{2}, \ldots, x^{10}$ be the points indicated in Figure 3.2. The three nongrid points are $x^{2}=(3 / 2,1 / 2)$, 


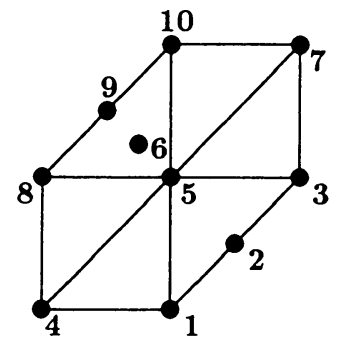

FIGURE 3.2

Domain $\Omega$ and location of interpolation points for Example 3.2.

$x^{6}=(3 / 4,5 / 4)$ and $x^{9}=(1 / 2,3 / 2)$. Ordering the box splines by diagonals and using the values in Figure 1.2, we obtain the following collocation matrix:

$$
A=\left(\begin{array}{ccc|cccc|ccc}
1 / 2 & 1 / 2 & 0 & 0 & 0 & 0 & 0 & 0 & 0 & 0 \\
1 / 8 & 6 / 8 & 1 / 8 & 0 & 0 & 0 & 0 & 0 & 0 & 0 \\
0 & 1 / 2 & 1 / 2 & 0 & 0 & 0 & 0 & 0 & 0 & 0 \\
\hline 0 & 0 & 0 & 1 / 2 & 1 / 2 & 0 & 0 & 0 & 0 & 0 \\
0 & 0 & 0 & 0 & 1 / 2 & 1 / 2 & 0 & 0 & 0 & 0 \\
0 & 0 & 0 & 0 & 1 / 4 & 1 / 4 & 0 & 1 / 32 & 7 / 16 & 1 / 32 \\
0 & 0 & 0 & 0 & 0 & 1 / 2 & 1 / 2 & 0 & 0 & 0 \\
\hline 0 & 0 & 0 & 0 & 0 & 0 & 0 & 1 / 2 & 1 / 2 & 0 \\
0 & 0 & 0 & 0 & 0 & 0 & 0 & 1 / 8 & 6 / 8 & 1 / 8 \\
0 & 0 & 0 & 0 & 0 & 0 & 0 & 0 & 1 / 2 & 1 / 2
\end{array}\right) .
$$

$A$ is singular since the middle diagonal block is singular.

Suppose we number the diagonals $\delta_{1}, \delta_{2}, \ldots, \delta_{d}$ in $\Omega$ from bottom right to top left. Thus, $\delta_{1}$ passes through the point $\left(n_{1}, k_{l}\right)$. (Cf. Figure 1.1). For $k=1,2, \ldots, d$, let

$$
D_{k}=\left\{x \in R^{2} \cap \Omega: \operatorname{dist}\left(x, \delta_{k}\right)<\sqrt{2} / 2\right\},
$$

where for $x \in R^{2}, S \subset R^{2}$, dist $(x, S)$ denotes the Euclidean distance from $x$ to the set $S$. The following theorem explains the results in Example 3.2.

THEOREM 3.1. Suppose $\Omega$ contains g grid points and d diagonals. Let

$$
\left\{x^{1}, \ldots, x^{g+d}\right\}=\left\{u^{1}, \ldots, u^{g}, v^{1}, \ldots, v^{d}\right\}
$$

where $u^{1}, \ldots, u^{g}$ are the grid points in $\Omega$ and $v^{1}, \ldots, v^{d}$ are points which satisfy

(i) $v^{k} \in D_{k}, k=1,2, \ldots, d$;

(ii) at most one of $v^{k}, v^{k+1}$ is in $D_{k} \cap D_{k+1}, k=1,2, \ldots, d-1$.

Then the $(1,1,2)$ box spline interpolation problem with points $x^{1}, \ldots, x^{g+d}$ is unisolvent if and only if $v^{k} \notin L_{k}, k=1,2, \ldots, d$, where $L_{k}$ is the collection of all straight lines with slope -1 through grid points on $\delta_{k}$.

Proof. We order $\left\{x^{1}, \ldots, x^{n}\right\}=\left\{u^{1}, \ldots, u^{g}, v^{1}, \ldots, v^{d}\right\}$ in $d$ groups. For $i=$ $1,2, \ldots, d$ group $i$ contains all grid points on diagonal $\delta_{i}$ plus $v^{i}$. Within each group we order from bottom left to top right, i.e., on the sum of the coordinates of the points. If the $d$-ordering is used for the basis functions then the collocation 
matrix $A$ will be block tridiagonal:

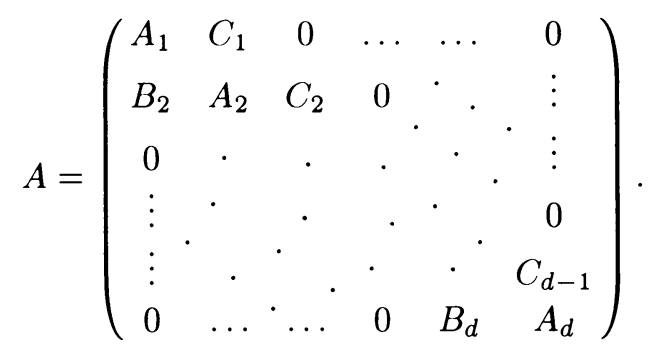

The matrices $B_{i}$ and $C_{i}$ contain at most one nonzero row, namely the one corresponding to $v^{i}$. If $v^{i}$ is on or above the $i$ th diagonal $\delta_{i}$, then $B_{i}=0$. Similarly, $C_{i}=0$ if $v^{i}$ is on or below the $i$ th diagonal $\delta_{i}$. Moreover, assumption (ii) implies that at most one of $C_{i-1}$ and $B_{i}$ can be nonzero. For if $C_{i-1}$ is nonzero, then $v^{i-1}$ is above $\delta_{i-1}$, thus $v^{i-1} \in D_{i-1} \cap D_{i}$. Also, if $B_{i}$ is nonzero, then $v^{i}$ is below $\delta_{i}$ and hence $v^{i} \in D_{i-1} \cap D_{i}$. But (ii) implies that at most one of $v^{i-1}$ and $v^{i}$ is in $D_{i-1} \cap D_{i}$. Hence either $C_{i-1}$ or $B_{i}$ must be zero as asserted.

It follows that $A$ is nonsingular if and only if all the diagonal blocks $A_{1}, A_{2}, \ldots, A_{d}$ are nonsingular.

Let, for some $i, 1 \leq i \leq d, p^{1}, \ldots, p^{k}$ be the interpolation points associated with $\delta_{i}$ and assume $v^{i}=p^{r}$. The matrix $A_{i}$ takes the following form, illustrated here for $k=10, r=4$,

$$
A_{i}=\left(\begin{array}{cc|ccc|ccccc}
1 / 2 & 1 / 2 & 0 & 0 & 0 & 0 & 0 & 0 & 0 & 0 \\
0 & 1 / 2 & 1 / 2 & 0 & 0 & 0 & 0 & 0 & 0 & 0 \\
\hline 0 & 0 & 1 / 2 & 1 / 2 & 0 & 0 & 0 & 0 & 0 & 0 \\
0 & 0 & a & b & c & 0 & 0 & 0 & 0 & 0 \\
0 & 0 & 0 & 1 / 2 & 1 / 2 & 0 & 0 & 0 & 0 & 0 \\
\hline 0 & 0 & 0 & 0 & 1 / 2 & 1 / 2 & 0 & 0 & 0 & 0 \\
0 & 0 & 0 & 0 & 0 & 1 / 2 & 1 / 2 & 0 & 0 & 0 \\
0 & 0 & 0 & 0 & 0 & 0 & 1 / 2 & 1 / 2 & 0 & 0 \\
0 & 0 & 0 & 0 & 0 & 0 & 0 & 1 / 2 & 1 / 2 & 0 \\
0 & 0 & 0 & 0 & 0 & 0 & 0 & 0 & 1 / 2 & 1 / 2
\end{array}\right),
$$

where $a, b$ and $c$ are the values of the basis functions which can be nonzero at $p^{r}$. It follows that $A_{i}$ is nonsingular if and only if the $3 \times 3$ middle block is nonsingular. This is equivalent to

$$
b-a-c \neq 0 .
$$

Now, $p^{r}=(u, v)$ can be in any of four types of triangles as illustrated in Figure 3.3. Thus, there are four cases to consider. Without loss of generality we assume that the origin is at the common vertex of the four triangles. Using Figure 3.1, we have

(i) $p^{r} \in T_{1}$. In this case, $a=\frac{1}{2}(u-1)^{2}-\frac{1}{2} v^{2}, b=\frac{1}{2}(v+1)^{2}-\frac{1}{2} u^{2}$, and $c=0$, so that $b-a-c=(v-u+1)(v+u)$. Thus, $b-a-c \neq 0$, except on the line $u+v=0$.

(ii) $p^{r} \in T_{2}$. Now we have $a=\frac{1}{2}(u-1)^{2}, b=1-\frac{1}{2}(v-1)^{2}-\frac{1}{2} u^{2}$, and $c=\frac{1}{2} v^{2}$. It follows that $b-a-c=\frac{1}{2}-\left(u-\frac{1}{2}\right)^{2}-\left(v-\frac{1}{2}\right)^{2} \neq 0$ for all $(u, v) \in T_{2}$.

(iii) $p^{r} \in T_{3}$. By symmetry we obtain the same value for $b-a-c$ as in case (ii).

(iv) $p^{r} \in T_{4}$. We can exchange $u$ and $v$ in case (i) to obtain $b-a-c=$ $(u-v+1)(u+v)$. Thus again, $b-a-c \neq 0$, except on the line $u+v=0$. 


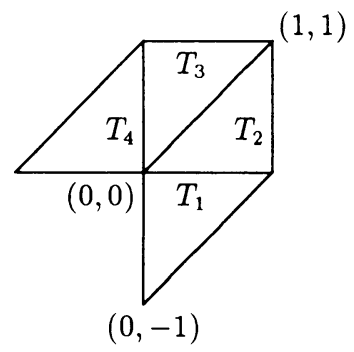

FIGURE 3.3

The four cases in the proof of Theorem 3.1 .

It follows that $A_{i}$ is nonsingular if and only if $v^{i}$ is not on the straight lines with slope -1 through the grid points of $\delta_{i}$. This completes the proof of the theorem.

We now examine the question of when the matrix $A$ is diagonally dominant. Since by (2.3) the elements in each row of $A$ are nonnegative and sum up to one, $A$ will be diagonally dominant if the diagonal element is greater than or equal to one half. For a $(1,1,2)$ box spline $B$ the set of all points $x \in R^{2}$ where $B(x)>\frac{1}{2}$ is shown as the region between the two dotted circular curves in the right half of Figure 1.2. Suppose $\Omega$ is as in Figure 1.1, and let $B_{1}, \ldots, B_{n}$ be the nonzero $(1,1,2)$ box splines on $\Omega^{0}$. Then the collocation matrix will be (weakly) diagonally dominant if $x^{i} \in \Omega$ are such that for $i=1,2, \ldots, n$

$$
x^{i} \in S_{i}=\left\{x \in \Omega: B_{i}(x) \geq \frac{1}{2}\right\} .
$$

For a general $\Omega$, points $x^{i}$ satisfying (3.2) will not always be distinct. Consider, for example, Figure 3.1. Here, $S_{1}=S_{2}=\{(1,0)\}$, and it is impossible to choose distinct $x^{1}$ and $x^{2}$ for diagonal dominance. The following theorem restricts $\Omega$ so that diagonal dominance is possible.

THEOREM 3.2. Let $\Omega$ be such that $k_{l}>0$ and $k_{u}>0$, and denote by $B_{1}, \ldots, B_{n}$ the box splines in $I(\Omega)$. Suppose $x^{1}, \ldots, x^{n}$ satisfy (3.2). Moreover, assume that for at least one point associated with each diagonal we have $x^{i} \in S_{i}^{0}=\{x \in \Omega$ : $\left.B_{i}(x)>\frac{1}{2}\right\}$. Then the $n \times n$ matrix $A=\left(B_{j}\left(x^{i}\right)\right)$ is nonsingular.

Proof. Since each $S_{i}$ only intersects one diagonal of $\Omega$, the matrix $A$ will be block tridiagonal of the form (3.1) if the $d$-ordering is used for $B_{1}, \ldots, B_{n}$. Moreover, each diagonal block $A_{i}$ is tridiagonal of the form

$$
A_{i}=\left(\begin{array}{cccccc}
1 / 2 & 1 / 2 & 0 & \ldots & \ldots & 0 \\
x & x & x & & & \vdots \\
0 & x & x & x & & \vdots \\
\vdots & & & & & 0 \\
\vdots & & & x & x & x \\
0 & \ldots & \ldots & 0 & 1 / 2 & 1 / 2
\end{array}\right) .
$$

Here, elements marked as $x$ are nonzero. The first and last row of $A_{i}$ must be of the form shown, since the first and last grid point on each diagonal must be an interpolation point. Furthermore, we observe that $A_{i}$ has at least 3 rows and columns, since $k_{l}>0$ and $k_{u}>0$. Finally we observe that $A_{i}$ is strictly diagonal 
dominant in at least one row. Thus, $A_{i}$ is an irreducible diagonally dominant matrix. By Theorem 1.8 in [12] the matrix $A_{i}$ is nonsingular. It now follows by a standard argument that $A$ itself is nonsingular. Indeed, we note that if all of the off-diagonal blocks contain at least one nonzero element, then $A$ will be an irreducible diagonally dominant matrix and hence nonsingular. Finally, if one or more of the off-diagonal blocks are zero, then $A$ is block triangular and we can test for nonsingularity by looking at each diagonal block in the block triangular form. Each of these diagonal blocks will in turn be block tridiagonal.

Analogous results could also be given for the quadratic box splines $(2,1,1)$ and $(1,2,1)$.

4. A Generalization. In this section we consider a Hermite interpolation problem for the box spline $(1,1, k)$, where $k \in N$ is given. This box spline is piecewise of degree $k$. It vanishes on all diagonals except one. Using (1.1) and the fact that for univariate B-splines

we have

$$
M_{k+1}(t \mid 0,1, \ldots, k+1)=\int_{0}^{1} M_{k}(t-u \mid 0,1, \ldots, k) d u
$$

$$
B^{(1,1, k)}(t, t)=M_{k+1}(t \mid 0,1, \ldots, k+1) .
$$

Thus, $B^{(1,1, k)}$ is equal to a univariate B-spline of order $k+1$ along the interior diagonal of its support.

Suppose $\Omega \subset R^{2}$ is given with $d$ diagonals $\delta_{1}, \delta_{2}, \ldots, \delta_{d}$. For the box spline with $m=(1,1, k)$ this is also the number of diagonals in $I(\Omega)$. If $p^{1}$ and $p^{2}$ are two points on $\delta_{i}$, we say that $p^{1}<p^{2}$ if $p^{1}$ is below $p^{2}$. Now, for $i=1,2, \ldots, d$, let

$$
x^{i, 1} \leq x^{i, 2} \leq \cdots \leq x^{i, \nu_{i}}
$$

be given points on $\delta_{i}$, where $\nu_{i}$ is the number of elements in $I(\Omega)$ which lie on $\delta_{i}$. Let $\phi_{i, j}, j=1,2, \ldots, \nu_{i}$, be the nonzero box splines on $\delta_{i}$ ordered from left to right. We define linear functionals $\lambda_{i, j}, j=1,2, \ldots, \nu_{i}$, by

$$
\lambda_{i, j} f=\max _{r}\left\{\left(D_{1}+D_{2}\right)^{r} f\left(x^{i, j}\right): x^{i, j-r}=x^{i, j}\right\},
$$

where $D_{1}$ and $D_{2}$ denote partial derivatives in the $x$ and $y$ direction, respectively. We then have

THEOREM 4.1. For $i=1,2, \ldots, d$, suppose that at most $k$ of the points $x^{i, 1}, \ldots$, $x^{i, \nu_{i}}$ are equal to one value. Then the above problem is unisolvent if and only if $\phi_{i, j}\left(x^{i, j}\right) \neq 0$ for all $i, j$.

Proof. We again use the $d$-ordering. Since each of the $\phi_{i, j}$ 's are nonzero only over one diagonal, the collocation matrix for the interpolation problem is block diagonal

$$
A=\operatorname{diag}\left(A_{1}, A_{2}, \ldots, A_{d}\right)
$$

where each diagonal block $A_{i}$ is a matrix of order $\nu_{i}$ with elements

$$
\lambda_{i, r} \phi_{i, s}, \quad r, s=1,2, \ldots, \nu_{i} .
$$

Now on each diagonal we have by (4.1) that $\phi_{i, 1}, \ldots, \phi_{i, \nu_{i}}$ are consecutive shifted univariate B-splines of order $k+1$. By Theorem 4.67 in [11], $A_{i}$ is nonsingular if and only if $\phi_{i, j}\left(x^{i, j}\right) \neq 0, j=1,2, \ldots, \nu_{i}$. 
5. Example. As seen in Table 3.1 , the $(1,1,2)$ box spline is a piecewise quadratic polynomial where the term $x y$ is missing. In the following example we interpolate points on the function $f(x, y)=x y$ using translates of the $(1,1,2)$ box spline as basis functions. The error in the approximation gives an indication of how well smooth functions can be approximated by $C^{0}$-quadratic box splines.

Example 5.1. Given $\Omega$ as in Figure 1.1, we interpolate $f(x, y)=x y$ on $h \Omega=$ $\{h x: x \in \Omega\}$ using a grid with spacing $h=1 / n$ and $h$-translates of the $(1,1,2)$ box spline. The interpolation points are on the diagonals midway between grid points. In addition, the end points of each diagonal are used. If $k_{l}=0$ or $k_{u}=0$, we also interpolate the end point derivative in the direction along the diagonal.

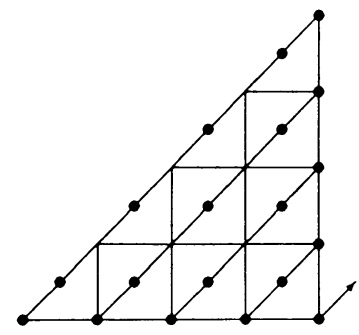

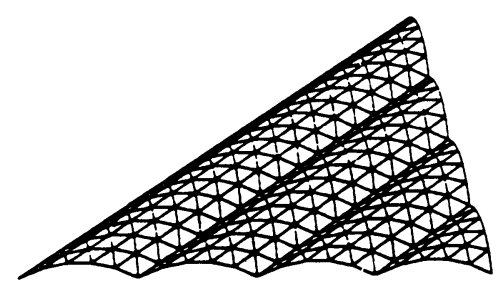

FIGURE 5.1

The interpolation points and the error in quadratic box spline interpolation to $f(x, y)=x y$ on a triangle.

The situation is illustrated for a triangle $\left(k_{l}=0\right)$ in Figure 5.1. By Theorem 4.1 the interpolation problem is unisolvent. The difference $f-g$, where $g$ is the interpolant, is also shown in Figure 5.1.

In this simple case we have

$$
\|f-g\|_{L^{\infty}(h \Omega)}=h^{2} / 8 .
$$

More specifically, on a square with length $h$ divided into two triangles $l$ and $u$ by an NE diagonal (cf. Figure 3.1) we have

$$
f(x, y)-g(x, y)= \begin{cases}h^{2}\left(z-z^{0}\right)\left(z^{0}+1-z\right) / 2 & \text { if }(x, y) \in l, \\ h^{2}\left(z-z^{0}\right)\left(z^{0}-1-z\right) / 2 & \text { if }(x, y) \in u\end{cases}
$$

where $z=(x-y) / h$ and $z^{0}$ is the difference between the $x$ and $y$ coordinate of the lower left corner of the square. (5.2) shows that the error is constant along diagonals. (5.1) follows immediately from (5.2). To show (5.2), we note that $f-g=0$ along each diagonal through grid points. This follows since we are doing quadratic spline interpolation to a quadratic along each diagonal. But then we can determine $g$ locally on each triangle. We can, for example, interpolate three points on the hypotenuse and the value and derivative in the diagonal direction at the right-angled corner. It can be verified directly that $g$ defined implicitly by (5.2) interpolates $f$ according to these interpolation conditions. Moreover, this $g$ is of the form of a quadratic in which the $x y$ term is missing,

$$
g(x, y)=a x^{2}+c y^{2}+d x+e y+f .
$$

By Table 1 , the $(1,1,2)$ box spline is precisely of this form on each triangle. This proves (5.2). 
In [6], Theorem 3.2 is applied to interpolate points on a pipe system. Points on circles and ellipses corresponding to bends on the pipe system are given. Interpolation points given at a bend correspond to points given on a diagonal in its respective domain $\Omega$, and a parametric $(1,1,2)$ box spline surface interpolates the points. Since the $(1,1,2)$ box spline is discontinuous in the first derivative across diagonals, the bend on the pipe system is reproduced.

Center for Industrial Research

Box 124

Blindern

0314 Oslo 3, Norway

Institutt for informatikk

University of Oslo

Box 1080

Blindern

0316 Oslo 3, Norway

1. C. DE BOOR \& K. HÖLlig, "Bivariate box splines and smooth pp functions on a three direction mesh," J. Comput. Appl. Math., v.9, 1983, pp. 13-28.

2. C. DE BOOR, "Multivariate approximation," in The State of the Art in Numerical Analysis (A. Iserles and M. J. D. Powell, eds.), Clarendon Press, Oxford, 1987, pp. 87-109.

3. E. W. Cheney, Multivariate Approximation Theory: Selected topics, CBMS-NSF Regional Conference Series in Applied Mathematics, vol. 51, SIAM, Philadelphia, Pa., 1986.

4. C. K. CHUi, T. X. HE \& R. H. WANG, "Interpolation of bivariate linear splines," in Alfred Haar Memorial Conference (J. Szabados and K. Tandori, eds.), North-Holland, Amsterdam, 1986.

5. C. K. CHUI \& T. X. HE, "On location of sample points for interpolation by bivariate $C^{1}$ quadratic splines," in Numerical Methods of Approximation Theory, vol. 8 (L. Collatz, G. Meinardus and G. Nürnberger, eds.), Birkhäuser, Basel, 1987, pp. 30-43.

6. M. DÆHLEN, "An example of bivariate interpolation with translates of $C^{0}$-quadratic boxsplines on a three direction mesh," Comput. Aided Geom. Des., v. 4, 1987, pp. 251-255.

7. K. HölliG, "Box splines," in Approximation Theory V (C. K. Chui, L. L. Schumaker and J. Ward, eds.), Academic Press, New York, 1986, pp. 71-95.

8. C. A. MiCChelli, "Algebraic aspects of interpolation," in Approximation Theory, Proc. Sympos. Appl. Math., vol. 36, Amer. Math. Soc., Providence, R.I., 1986, pp. 81-102.

9. T. I. MUeller, Geometric Modelling with Multivariate B-splines, Dissertation, Dept. of Comp. Sci., Univ. of Utah, 1986.

10. K. JetTER, "A short survey on cardinal interpolation by box splines," in Topics in Multivariate Approximation (C. K. Chui, L. L. Schumaker and F. Utreras, eds.), Academic Press, New York, 1987, pp. 125-139.

11. L. L. SChUMAKER, Spline Functions: Basic Theory, Wiley, New York, 1981.

12. R. S. VARGA, Matrix Iterative Analysis, Prentice-Hall, Englewood Cliffs, N.J., 1962. 